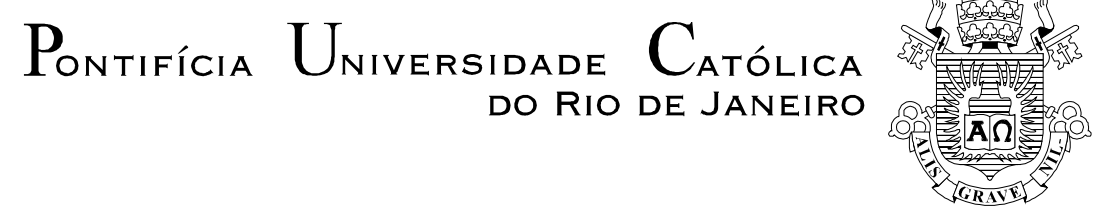

Cristian Enrique Muñoz Villalobos

Paralelização Heterogênea da Programação Genética Linear com Inspiração Quântica

Dissertação de Mestrado

Dissertação apresentada como requisito parcial para obtenção do título de Mestre pelo Programa de PósGraduação em Engenharia Elétrica da PUC-Rio.

Orientador: Prof. Marco Aurélio Pacheco Cavalcanti Co-Orientador: Prof. Douglas Mota Dias

Rio de Janeiro

Abril de 2014 


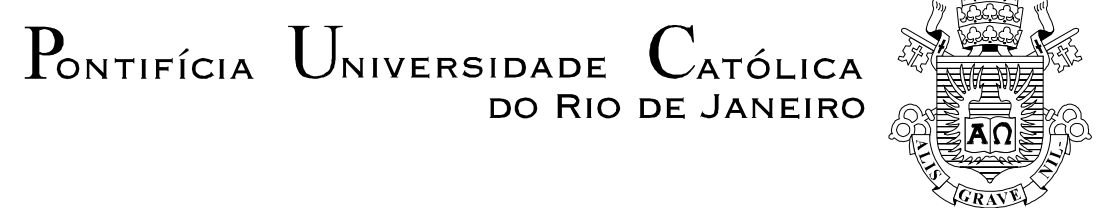

Cristian Enrique Muñoz Villalobos

\section{Paralelização Heterogênea da Programação Genética Linear com Inspiração Quântica}

Dissertação apresentada como requisito parcial para obtenção do título de Mestre pelo Programa de PósGraduação em Engenharia Elétrica da PUC-Rio. Aprovada pela Comissão Examinadora abaixo assinada.

Prof. Marco Aurélio Pacheco Cavalcanti

Orientador

Departamento de Engenharia Elétrica - PUC-Rio

Prof. Douglas Mota Dias

Co-orientador

Departamento de Engenharia Elétrica - PUC-Rio

Prof. Karla Tereza Figueiredo Leite

Departamento de Engenharia Elétrica - PUC-Rio

Prof. Noemi de la Roque Rodriguez

Departamento de Informática - PUC-Rio

Prof. Marley Maria Bernardes Rebuzzi Vellasco

Departamento de Elétrica - PUC-Rio

Prof. José Eugênio Leal

Coordenador Setorial do Centro Técnico Científico - PUC-Rio

Rio de Janeiro, 04 de abril de 2014 
Todos os direitos reservados. É proibida a reprodução total ou parcial do trabalho sem autorização da universidade, do autor e do orientador.

\section{Cristian Enrique Muñoz Villalobos}

Formado em Engenharia Mecatrônica pela Universidad Nacional de Ingeniería - UNI, Lima, Peru (2005-2010).

Ficha Catalográfica

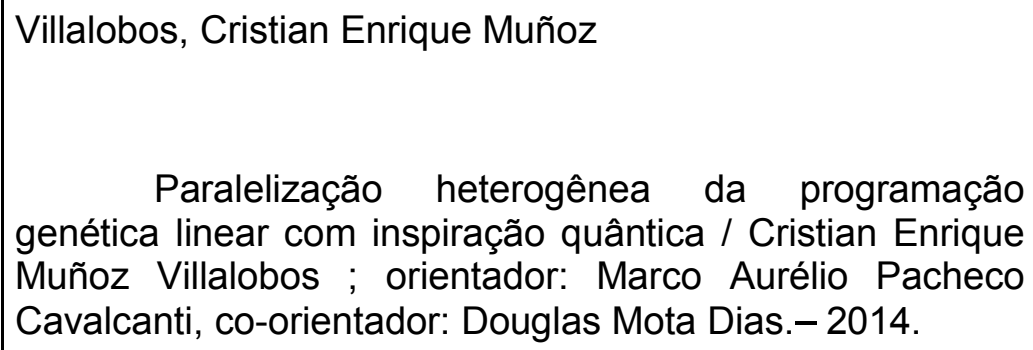

Paralelização heterogênea da programação genética linear com inspiração quântica / Cristian Enrique Muñoz Villalobos ; orientador: Marco Aurélio Pacheco Cavalcanti, co-orientador: Douglas Mota Dias.- 2014.

82 f. ; $30 \mathrm{~cm}$

Dissertação (mestrado) - Pontifícia Universidade Católica do Rio de Janeiro, Departamento de Engenharia Elétrica, 2014.

Inclui bibliografia.

1. Engenharia elétrica - Teses. 2. Paralelização heterogênea. 3. Programação genética linear. 4. Algoritmos evolutivos com inspiração quântica. 5. Computação de alto desempenho. 6. Computação com GPU. 7. Visualização gráfica. I. Cavalcanti, Marco Aurélio Pacheco. II. Dias, Douglas Mota. III. Pontifícia Universidade Católica do Rio de Janeiro. Departamento de Engenharia Elétrica. IV. Título. 


\section{Agradecimentos}

A Deus.

Ao meu orientador Professor Marco Aurélio, pelo apoio e parceria para a realização deste trabalho.

Ao meu coorientador Douglas, pela confiança, estímulo e importantes contribuições.

Aos meus pais, Luisa e José, pela educação, atenção e carinho.

Aos meus irmãos Jannet e Pepito, por todo apoio, paciência e compreensão.

À Frances Blank e Juan Lazo, amigos da PUC-Rio.

Aos Professores. Ricardo Rodríguez Bustinza e Elizabeth Villota Cerna, da

Universidade Nacional de Engenharia, pelo apoio para começar meus estudos de pós-graduação na PUC-Rio.

A todos os amigos e familiares que de uma forma ou de outra me estimularam e me ajudaram.

Aos professores que participaram da Comissão examinadora.

Ao CNPq, à PUC-Rio e à FAPERJ pelos auxílios concedidos, sem os quais este trabalho não poderia ter sido realizado. 


\section{Resumo}

Muñoz, Cristian Enrique Villalobos. Pacheco, Marco Aurélio Cavalcanti; Dias, Douglas Mota. Paralelização Heterogênea da Programação Genética Linear com Inspiração Quântica. Rio de Janeiro, 2014. 82p. Dissertação de Mestrado - Departamento de Engenharia Elétrica, Pontifícia Universidade Católica do Rio de Janeiro.

Um dos principais desafios da ciência da computação é conseguir que um computador execute uma tarefa que precisa ser feita, sem dizer-lhe como fazê-la. A Programação Genética (PG) aborda este desafio a partir de uma declaração de alto nível sobre "o que é necessário ser feito" e cria um programa de computador para resolver o problema automaticamente. Nesta dissertação, é desenvolvida uma extensão do modelo de Programação Genética Linear com Inspiração Quântica (PGLIQ) com melhorias na eficiência e eficácia na busca de soluções. Para tal, primeiro o algoritmo é estruturado em um sistema de paralelização heterogênea visando à aceleração por Unidades de Processamento Gráfico e a execução em múltiplos processadores CPU, maximizando a velocidade dos processos, além de utilizar técnicas otimizadas para reduzir os tempos de transferências de dados. Segundo, utilizam-se as técnicas de Visualização Gráfica que interpretam a estrutura e os processos que o algoritmo evolui para entender o efeito da paralelização do modelo e o comportamento da PGLIQ. Na implementação da paralelização heterogênea, são utilizados os recursos de computação paralela como Message Passing Interface (MPI) e Open Multi-Processing (OpenMP), que são de vital importância quando se trabalha com multi-processos. Além de representar graficamente os parametros da PGLIQ, visualizando-se o comportamento ao longo das gerações, uma visualização 3D para casos de robôtica evolutiva é apresentada, na qual as ferramentas de simulação dinâmica como Bullet SDK e o motor gráfico OGRE para a renderização são utilizadas.

\section{Palavras-chave}

Paralelização heterogênea; programação genética linear; algoritmos evolutivos com inspiração quântica; computação de alto desempenho; computação com GPU; visualização gráfica. 


\section{Abstract}

Muñoz, Cristian Enrique Villalobos. Pacheco, Marco Aurélio Cavalcanti (Advisor); Dias, Douglas Mota (Co-Advisor). Heterogeneous Parallelization of Quantum-Inspired Linear Genetic Programming. Rio de Janeiro, 2014. 82p. MSc. Dissertation - Departamento de Engenharia Elétrica, Pontifícia Universidade Católica do Rio de Janeiro.

One of the main challenges of computer science is to get a computer execute a task that must be done, without telling it how to do it. Genetic Programming (GP) deals with this challenge from a high level statement of "what is needed to be done" and creates a computer program to solve the problem automatically. In this dissertation we developed an extension of Quantum-Inspired Linear Genetic Programming Model (QILGP), aiming to improve its efficiency and effectiveness in the search for solutions. For this, first the algorithm is structured in a Heterogeneous Parallelism System, Aiming to accelerated using Graphics Processing Units GPU and multiple CPU processors, reducing the timing of data transfers while maximizing the speed of the processes. Second, using the techniques of Graphic Visualization which interpret the structure and the processes that the algorithm evolves, understanding the behavior of QILGP. We used the highperformance features such as Message Passing Interface (MPI) and Open MultiProcessing (OpenMP), which are of vital importance when working with multiprocesses, as it is necessary to design a topology that has multiple levels of parallelism to avoid delaying the process for transferring the data to a local computer where the visualization is projected. In addition to graphically represent the parameters of PGLIQ devising the behavior over generations, a 3D visualization for cases of evolutionary robotics is presented, in which the tools of "dynamic simulation" as Bullet SDK and graphics engine OGRE for rendering are used. This visualization is used as a tool for a case study in this dissertation.

\section{Keywords}

Heterogeneous parallelization; linear genetic programming; quantum inspired evolutionary algorithms; high performance computing; GPU computing; graphic visualization. 


\section{Sumário}

1 . Introdução 12

1.1. Motivações 12

1.2. Objetivos 14

1.3. Descrição do Trabalho 15

1.4. Organização do Trabalho 16

2. Fundamentação Teórica 18

2.1. Introdução 18

2.2. Computação Evolutiva 18

2.2.1. Programação Genética Linear 19

2.2.2. Demes 20

2.3. Programação Genética Linear com Inspiração Quântica (PGLIQ) 21

$\begin{array}{ll}\text { 2.3.1. Plataforma } & 21\end{array}$

2.3.2. Representação 22

2.3.3. Indivíduo Clássico 22

2.3.4. Indivíduo Quântico 23

2.3.5. Programas Evoluídos 24

2.3.6. Avaliação de um Indivíduo Clássico 24

2.3.7. Operador Quântico 24

2.3.8. Estrutura e Funcionamento do Modelo 25

2.4. Computação de Alto Desempenho 28

2.4.1. Introdução: GPUs vs CPUs 28

2.4.2. Conceitos gerais de computação paralela 29

2.4.3. Modelos de programação paralela utilizada 32

2.4.4. Ferramentas de programação paralela utilizadas 32

2.4.5. Níveis de Paralelismo 37

2.4.6. Torque 39

2.5. Visualização Gráfica $\quad 40$

2.5.1. Visualização de Dados Técnicos 40

2.5.2. Visualização em Computação Evolucionária 40 
3. Implementação do Sistema 44

3.1. Paralelização em CUDA (GPU-PGLIQ) 44

3.2. Paralelização com OpenMPI (GPU-PGLIQ) 46

3.3. Paralelização com OpenMP (GPU-PGLIQ) 48

3.3.1. Paralelização Hibrida MPI /OpenMP (GPU-PGLIQ) 49

3.4. Visualização Gráfica 53

3.4.1. Visualização do Melhor Individuo Quântico 54

3.4.2. Visualização da estrutura de Sub-rotinas 54

3.4.3. Simulação e Animação 54

3.5. Análise da PGLIQ 55

4. Estudo de Casos 61

4.1. Configuração 61

4.2. Distância Euclidiana 62

4.2.1. Avaliação de Desempenho: 65

4.3. Chapéu Mexicano 66

4.3.1. Avaliação de Desempenho 68

4.4. Estudo de Caso: Robótica Evolutiva 69

4.4.1. Configuração 69

4.4.2. Evolução da caminhada do robô.

5. Conclusões e Trabalhos Futuros $\quad 77$

5.1. Conclusões $\quad 77$

$\begin{array}{ll}\text { 5.2. Trabalhos Futuros } & 78\end{array}$

$\begin{array}{ll}\text { 6. Referências bibliográficas } & 79\end{array}$ 


\section{Lista de Figuras}

Figura 1. Representação gráfica de métodos algorítmicos e

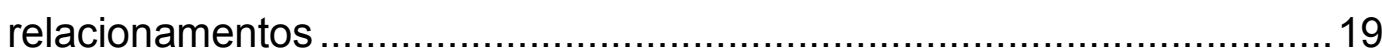

Figura 2. Representação típica de PG linear para programas. ................20

Figura 3. Modelo de demes como anéis (à esquerda) e rede de

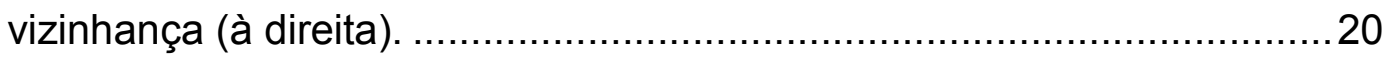

Figura 4. Cromossomo de um indivíduo clássico..................................23

Figura 5. Diagrama descritivo básico do modelo PGLIQ........................26

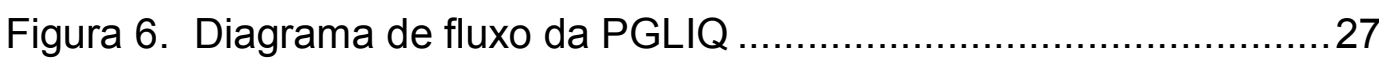

Figura 7. Comparação de desempenho de GPUs vs CPU.

Desempenho da GPU continua a aumentar a um ritmo muito rápido. .....28

Figura 8. Arquiteturas de sistema de multiprocessadores.......................30

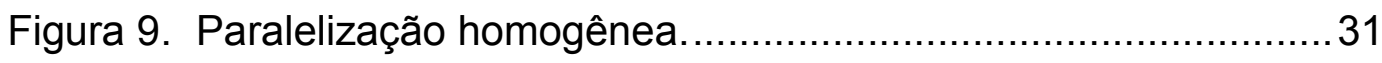

Figura 10. Paralelização heterogênea................................................ 31

Figura 11. Aplicação conjunta CPU-GPU............................................ 33

Figura 12. (a) Streaming Processors e (b) Streaming Multiprocessor ......33

Figura 13. Modelo MPI. (a) Grupos MPI. (b) Comunicadores MPI. ..........35

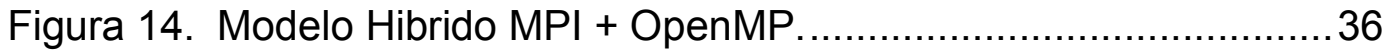

Figura 15. Algoritmo de Programação Híbrida …………………........... 37

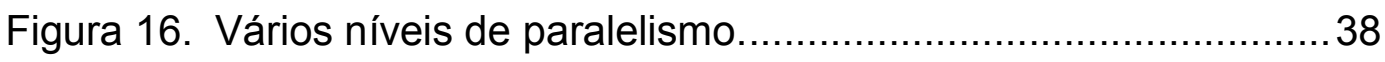

Figura 17. Gerenciador de Tarefas Torque ………............................... 39

Figura 18. Técnica Embodied Evolution ............................................. 42

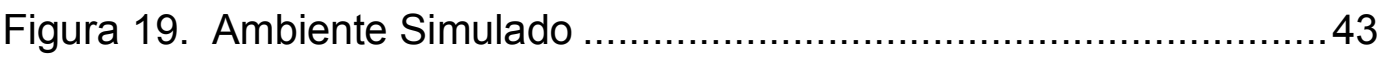

Figura 20. Kernels utilizados em cada geração pela GPU-PGLIQ ..........45

Figura 21. Porcentagem de tempo de processamento de cada kernel

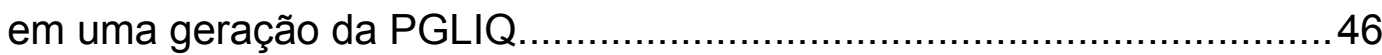

Figura 22. Estrutura da evolução com demes utlizando CUDA e OpenMPI

Figura 23. Topologia Multi-GPUs com memória compartilhada no Host. 48

Figura 24. Estrutura Base MPI/OpenMP ….......................................... 49

Figura 25. Agrupamentos dos nós dos clusters. ..................................50 
Figura 26. Topologia MPI para Algoritmo Evolutivos .............................53

Figura 27. Distribução de probabilidades da PGLIQ .............................56

Figura 28. Estrutura nova etapa de Reset.........................................57

Figura 29. Etapas de Reset. (a) Reset padrão. (b) reset proposto...........58

Figura 30. Estrutura de sub-rotinas da PGLIQ ....................................59

Figura 31. Gráfico evolutivo do estudo de caso "Distância Euclideana"

em função do tempo médio de cada execução dos modelos....................62

Figura 32. Variação em relação à aptidão AIMGP em porcentage". .......63

Figura 33. Comparação Gráfica de desempenhos obtidos pelos demes no estudo de caso "Distância Euclideana".....................................64

Figura 34. Gráfico evolutivo do estudo de caso "Chapéu Mexicano" em função do tempo médio de cada execução dos modelos....................66

Figura 35. Variação em relação à aptidão AIMGP em porcentage. ........67

Figura 36. Comparação Gráfica de desempenho obtido pelos demes no estudo de caso "Chapéu Mexicano"......................................................68

Figura 37. Robô Hexápode no ambiente de simulação BULLET .............69

Figura 38. Paralelismo Heterogêneo da PGLIQ para evolução do

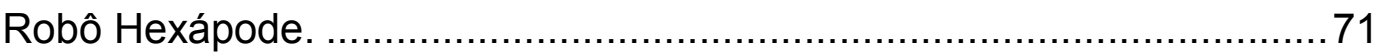

Figura 39. Sistema de evolução do hexápode .....................................72

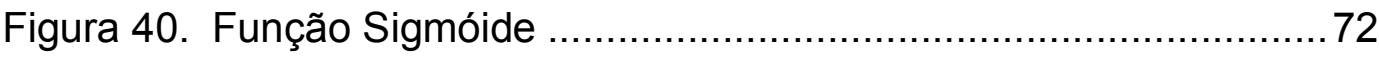

Figura 41. Gráfico evolutivo do estudo de caso "Robótica Evolutiva" em função do número médio de indivíduos avaliados por

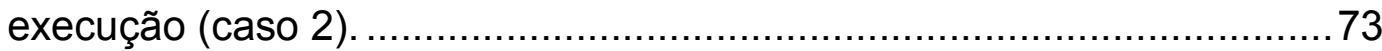

Figura 42. Salto realizado pelo hexápode .............................................. 73

Figura 43. Caminhar robô hexápode...................................................75 


\section{Lista de Tabelas}

Tabela 1. Exemplo de um conjunto de funções e seus tokens ................23

Tabela 2. Configuração do modelo PGLIQ para os experimentos .........61 61

Tabela 3. Comparação de média e desvio padrão das aptidões dos melhores indivíduos por geração para o estudo de caso

"Distância Euclideana".

Tabela 4. Comparação do número de acertos obtidos no estudo de caso "Distância Euclideana".

Tabela 5. Comparação da análise com demes obtidos no estudo de caso "Distância Euclideana".

Tabela 6. Comparação de Tempos para o caso de Estudo de

Distância Euclidiana

Tabela 7. Comparação de média e desvio padrão das aptidões dos melhores indivíduos por geração para o estudo de caso "Chapéu Mexicano" e Variação em Relação.

Tabela 8. Comparação do número de acertos obtidos no estudo de caso "Chapéu Mexicano".

Tabela 9. Comparação de Tempos para o caso de Estudo de

Chapéu Mexicano 68

Tabela 10. Tabela de Resultados de experimentos utilizando 24 CPUs. 74 Tabela 11. Resultados de experimentos utilizando o número máximo de CPUs (84). 